\title{
Simulation of Peer-to-peer streaming over large-scale networks using OPSS
}

\author{
Lorenzo Bracciale \\ DIE \\ University of Rome "Tor \\ Vergata", Italy
}

\author{
$\underset{\text { DIE }}{\text { Francesca Lo Piccolo }}{ }^{\dagger}$ \\ University of Rome "Tor \\ Vergata", Italy \\ Stefano Salsano \\ University of Rome "Tor \\ Vergata", Italy
}

\author{
$\underset{\text { DIE }}{\text { Dario Luzzi }}$ \\ University of Rome "Tor \\ Vergata", Italy
}

\begin{abstract}
In this paper we present OPSS, an Overlay Peer-to-peer Streaming Simulator designed to simulate a large scale (i.e. in the order of $100 \mathrm{~K}$ nodes) peer-to-peer streaming systems. OPSS is able to simulate a fair (i.e."TCP-like") sharing of the uplink and downlink bandwidth among different connections, and it guarantees extensibility by allowing the implementation of different peer-to-peer streaming algorithms as separate modules. Therefore it allows to simulate the behavior of arbitrary tree-based or mesh-based approaches. In particular, we implemented two trivial tree-based and meshbased approaches for which we could easily find an analytic model of chunk distribution delay. The results of the model have been compared with the simulation output, showing an excellent fit. Source code of OPSS is available under the GPL license ${ }^{1}$.
\end{abstract}

\section{Categories and Subject Descriptors}

C.2.4 [Distributed Systems]: Distributed applicationsP2P streaming systems; C.4 [Performance of Systems]: Performance attributes; I.6.8 [Types of Simulation]: Discrete event

\section{General Terms}

Performance ${ }^{*}$ lorenzo.bracciale@uniroma2.it
${ }^{\dagger}$ francesca.lopiccolo@uniroma2.it
${ }^{\ddagger}$ dario.luzzi@uniroma2.it
$\S_{\text {stefano.salsano@uniroma2.it }}$
${ }^{1}$ An abstract on the same topic will appear in a special issue
of Performance Evaluation Review.

Permission to make digital or hard copies of all or part of this work for personal or classroom use is granted without fee provided that copies are not made or distributed for profit or commercial advantage and that copies bear this notice and the full citation on the first page. To copy otherwise, to republish, to post on servers or to redistribute to lists, requires prior specific permission and/or a fee.

NSTools '07, October 22, 2007, Nantes, France

Copyright 2007 ICST 978-963-9799-00-4.

\section{Keywords}

Simulation of large-scale networks, performance metrics

\section{INTRODUCTION}

Even if IP multicast has originally been introduced with the purpose of offering point-to-multipoint content distribution services, many deployment issues have still to be solved. As argued in [1], IP multicast calls for multicast-capable routers able to maintain per group state information, which seriously limit its scalability. Second, IP multicast is a best effort service, and providing higher level features such as reliability, congestion control, flow control, and security has been shown to be more difficult than in the unicast case. Finally, IP multicast requires changes at the infrastructure level, and this slows down the deployment pace.

Due to this, more and more researchers are investigating application level multicast as solution to stream multimedia audio and video content from a source to a large number of end users. This approach consists of end hosts, which according to peer-to-peer $(\mathrm{P} 2 \mathrm{P})$ paradigm auto-organize themselves in an overlay network out of unicast tunnels across participating overlay nodes. Relaying data among overlay nodes allows then the multicast service.

Overlay multicast distribution trees represent the most natural way of extending IP level multicast to application level. To name a few, NARADA [2], HMTP [3], NICE [4], ZIGZAG [5], CoopNet [6], SplitStream [7] are tree-based P2P streaming systems. However, while tree-based topologies are well suited to dedicated IP multicast routers, they could suffer from re-configurability problems in presence of the high churn rate of $\mathrm{P} 2 \mathrm{P}$ nodes. In reason of this, overlay mesh-based and unstructured topologies have also been proposed. CoolStreaming/DONet [8] and GridMedia [9] offer examples of the latter approach.

The performance analysis of a large scale real-time streaming system is far from trivial. One has to consider both the "control plan" related aspects (how peers exchange the information about chunk availability and schedule the transmission of chunks) and the "transport plane" related aspects (how much time is necessary to transmit chunks given the available transmission resources on the access and backbone links and considering the competition among different peers). 
The paper is organized as follows. In section 2 we review the current approaches of performance evaluation of realtime P2P streaming systems and we highlight their limitations. In section 3 we present OPSS [10], a simulator designed to simulate a fair (i.e. "TCP-like") sharing of the uplink and downlink bandwidth among different connections in large scale (i.e. in the order of $100 \mathrm{~K}$ nodes) P2P streaming systems. In section 4, OPSS is used to provide a simulative evaluation of two real-time streaming algorithms. An analytical performance analysis of the algorithms is also given. Note that the purpose of this section is neither the proposal of a specific algorithm nor the comparison of a set of algorithms. We rather aim at showing that OPSS is a valuable tool for performance analysis and we show this by looking at the excellent matching between the simulative performance analysis and the analytic results. Finally, section 5 concludes the paper.

\section{REVIEW OF EXISTING WORKS}

In this section we first briefly introduce some existing systems for P2P live media streaming and we focus especially on the existing works that try to evaluate their performance. Then we analyze a set of existing simulators for P2P systems.

CoolStreaming/DONet [8] is a Data-driven Overlay Network for live media streaming. The overlay membership management is built on a gossip-based protocol [11]. In gossip-based protocols, a node sends a newly generated message to a set of randomly selected nodes; these nodes do similarly in the next round, and so other nodes do until the message is spread. The video stream is divided into segments or chunks, and chunk availability in the node buffer is represented by a Buffer Map (BM), where bit 1 and 0 indicate that a segment is respectively available and unavailable. Each node learns about chunk availability by periodically exchanging its BM with the BMs of its overlay neighbors, also called partners. DONet is built on a "pull" approach, i.e. the download of a given chunk starts only if a node requests that chunk from a supplier partner. The performance of DONet has been evaluated in [8] using PlanetLab [12][13]. PlanetLab is a global overlay network to support the design and the performance evaluation of applications widely distributed over the Internet. The control overhead and the continuity index are considered as performance metrics. The former represents the ratio between the control traffic volume and the video traffic volume; the latter is the number of segments that arrive before or on playback deadlines over the total number of segments. DONet performance is also compared with the performance of a tree-based overlay streaming system. Besides the continuity index, the average hop count is considered as raw approximation of end-toend delay for delivering each segment. The number of used PlanetLab nodes ranges from 10 to 200 (passing through $50,100,150)$.

GridMedia [9] is a unstructured P2P live media streaming system which tries to overcome the limitation of the DONet pull approach. It is based on a "push-pull" approach that implies requesting stream packets in pull mode at start up and having nodes relaying stream packets without explicit request in the immediate following phase. PlanetLab testbed is used to evaluate GRidMedia performance in [9]. Pull and push-pull approaches are compared. The proposed experimental results relate to a number of PlanetLab nodes ranging from 300 to 340 . Among the proposed performance indexes, we mention i) the absolute delay, that is the delay between the sampling time at the server and the playback time at the local node; ii) the delivery ratio, that is the ratio between the number of stream packets arriving before or right on absolute playback deadline and the total number of packets; iii) the $\alpha$-playback-time, that is the minimum absolute delay at which the delivery ratio is larger than $\alpha$ $(0 \leq \alpha \leq 1)$; iv) the control overhead of the gossip protocol, that is the average ratio between the control traffic and the total traffic at each node.

The same authors as [9] focus in [14] on the optimal streaming scheduling problem in data-driven overlay networks. The optimal streaming scheduling problem aims at addressing how each node optimally decides from which neighbor to request which block, and how it allocates its limited outbound bandwidth to every neighbor, in order to maximize the throughput. This scheduling problem is formulated as a classical min-cost network flow problem and two resolution strategies are considered. The first one is a global optimal solution which assumes a centralized knowledge of all network states, the second one is an heuristic procedure which is fully distributed and calls for only local information exchange. To validate their algorithm, they use a discrete event-driven $\mathrm{P} 2 \mathrm{P}$ simulator to simulate a data driven overlay network of 500 nodes. However, the authors do not give details about the kind of simulator they use. The low number of simulated nodes makes the hypothesis of packet-level simulator reasonable. The considered metric is the average delivery ratio, that is the ratio between the number of packets arriving before or right on the playback deadline averaged on all the nodes and the total number of packets. The proposed solution is compared with DONet, ChainSaw [15] and round-robin streaming scheduling.

NICE [4] is another overlay P2P live streaming system, but it is built on a hierarchically connected overlay topology differently from the previously described systems. The host hierarchy is used to define different overlay structures for control messages and data delivery paths. End-to-end latency is used as distance metric between host and it drives the association of nodes in clusters. NICE clusters and layers are created, maintained and eventually repaired by a fully distributed protocol. Data overlay delivery path is instead the tree rooted at data source and implicitly defined by the control overlay topology hierarchy. A packet level simulator is used to evaluate NICE performance. Network topologies are generated using the Transit-Stub graph model and the GT-ITM topology generator [16]. The number of end hosts in the multicast group is ranges from 8 to 2048 . Performance metrics such as the average link stress and the average path length are investigated. The first one is the number of identical packets sent over each underlying network link averaged across all the network links. The second one is the length (in number of hops) of the path from the source to the hosts averaged across all the hosts. The fraction of members that correctly receive the data packets in case of node failures and the byte-overhead for control traffic at the access links of the end-hosts are evaluated too. The achieved results are compared with the results obtained by simulating the application-layer multicast protocol Narada [2].

There are other P2P streaming applications like PPLive [17] or Sopcast [18] that are widely deployed but whose al- 
gorithms are not under public domain. The only solution for investigating their performance and behavior is to use a black-box measurement-based approach, as in [19] and in [20].

Regardless of the P2P live streaming systems described so far, a large number of $\mathrm{P} 2 \mathrm{P}$ simulators has recently emerged. Most of them mainly focuses on simulating the resource search phase and the related query message handling. This is the case of Aurora [21] and Serapis [22], which model the key announcement, insert or request process of Freenet-like systems. Similarly, P2Psim [23], FreePastry [24] and the Chord simulator [25], simulate only the DHT-based (Distributed Hash Table) search phase. A similar approach is employed in other general-purpose $\mathrm{P} 2 \mathrm{P}$ simulators, such as Neurogrid [26][27], 3LS [28], and Peersim [29][30]. Although the $\mathrm{P} 2 \mathrm{P}$ query/search phases are undoubtedly representative of a $\mathrm{P} 2 \mathrm{P}$ system, there is plenty of interest in quantitatively characterizing performance figures related to the resource distribution process among involved peers. All the previously mentioned simulation platforms are not suitable to this purpose, as they neglect the process of distributing data across peers. In fact, with regard to the low level network dynamics, either their effect is totally neglected, as in [21] and [26], where the overlay message transmission is immediate, or an exponentially distributed packet delay is used, as in [25], or the concept of distance between any two nodes is somehow defined and the overlay message transmission time is identified with the latency between the relative nodes, as in [22][23][24][28][29].

To properly model the data distribution phase, GnutellaSim [31][32] interfaces with the ns-2 [33] discrete event packet-based network simulator, which provides a very detailed packet-level simulation model of the underlying transport network. However, such a simulation model compromises the scalability of the resulting simulation, as only a few hundreds nodes may be properly simulated in reasonable time with such a level of details.

\section{SIMULATOR DESCRIPTION}

\subsection{Objective}

The review of existing research works on the topic of $\mathrm{P} 2 \mathrm{P}$ streaming has pointed out that it is possible to identify three different approaches of performance evaluation: i) measurement-based studies or real systems ii) experimental testbeds, such as PlanetLab, and iii) simulation tools. Measurement-based studies do not allow to consider different alternatives and to evaluate performance in advance of building and deploying a system. Experimental testbeds and current simulation tools suffer from scalability problems for different reasons. On the one hand, experimental testbeds would require a large network of emulator nodes, which is not easy to realize and to manage. On the other hand, the current simulation tools either are mostly oriented to the search phase and neglect the content distribution phase or perform the simulation at packet level, making unpractical to simulate a P2P live streaming system over a network of the order of $100 \mathrm{~K}$ peers. Typical available results concern network size of the order of hundreds or few thousands peers. This is not representative of real-life $\mathrm{P} 2 \mathrm{P}$ video streaming systems, which aim at streaming live multimedia content to a very large number, several hundred thousands if not millions, of users. It may be the case that network dynamics simulated in small-scale networks are not representative of large-scale P2P system deployments.

On basis of the above observation, we propose OPSS [10], a new simulative approach that makes P2P video streaming performance evaluation scalable.

\subsection{How does OPSS achieve scalability?}

In order to circumvent the tight scalability limits imposed by packet-based simulators and simultaneously to model networks dynamic with acceptable accuracy level, OPSS was conceived as discrete-event fluid-flow simulator. This allows to simulate the data distribution at the flow level, i.e. neglecting transmissions of single packets and focusing on events, such as start/end of a file or a file chunk transmission, which lead to a variation in the rate of the connections among peers. This approach dramatically reduces the number of simulation events and the related memory and computational load with respect to packet-level simulation, while retaining a satisfactory accuracy in the model of the data delivery process. We also assume that all active connections share fairly the available transmission resources, as it happens if peer nodes use TCP as transport protocol and round trip times are of the same order of magnitude. These assumptions justify the meaning of "TCP-like" sharing of the uplink and downlink bandwidth among different connections. Under this hypothesis it is possible to use a max-min fair [34] rate allocation algorithm in order to evaluate the available capacity for each connection, given the link bandwidth constraints. The notion of max-min fair allocation is based on the following premises: i) no entity should receive an allocation larger than its demand, and ii) increasing the allocation of any entity should not result in the decrease of the allocation of another entity that received an equal or smaller allocation. It well approximates the TCP-like sharing uploading and downloading bandwidth between concurrent flows.

Evaluating the max-min fair rate allocation in a network of hundred of thousand peers, with millions of active connections is not an easy task. The classical centralized implementation of max-min fair rate allocation (as suggested for instance in [34]) does not scale well for the network dimensions of our interest. The overall computational load of max-min fair allocation in our scenario is the product of two different factors. On the one hand, a max-min fair recomputation is required every time a new traffic relation is established, or an old traffic relation is completed or interrupted (e.g. because of peer disconnection), the frequency of these events being linearly dependent on the number of simulated peers ${ }^{2}$. On the other hand, the implementation suggested in [34] requires to re-compute the allocated rates per each network node, and thus it results in a complexity which grows linearly with the number of simulated peers.

\footnotetext{
${ }^{2}$ In fact, given a peer connected to the network with an access link of capacity $C$ byte/second and uploading/downloading a number of files with size $L$ bytes according to a processor-sharing queuing discipline, the average service rate is (assuming that all the link capacity is used) $C / L$ file/second. Since a re-computation of the max-min fair rate allocation algorithm is required at each change in the served traffic relations, given $N$ nodes, the number of recomputations per second is approximated by $N \cdot C / L$, hence linearly dependent on the network size. Note that we have neglected the fact that new arriving requests, if not queued, add further re-computation events.
} 
To the best of our knowledge, existing P2P fluid-flow simulators, such as [35] or [36], never rely on the exact implementation of the max-min fair rate allocation algorithm, but approximate it to improve its computational effectiveness. For example, [35] and [36] rely on an approach called (in [35]) minimum-share allocation. The rate allocated to a connection is given by the minimum among the fair share rates (link bandwidth divided by number of connections) at each involved link. Clearly, the resulting computational efficiency is traded off with an underestimation of the bandwidth actually used by flows: the excess bandwidth which a connection bottlenecked at a remote node makes available at a node, is not exploited by the other connections. Numerical results presented in [35] show a difference less than $10 \%$ in terms of completion time for file transfers with respect to the ns-2 computation. However, [35] does not mention whether heterogeneous access links are employed in their simulation results. Indeed, we intuitively expect that the accuracy of such an approximation might notably diminish in a highly bandwidth-heterogeneous scenario and that, despite its computational weight, an exact max-min fair bandwidth allocation algorithm should be employed in fluid-flow simulators.

Due to this, we have developed OPSS starting from the exact and efficient implementation of the max-min fair rate allocation algorithm proposed in [37]. As it was observed in [37], when a new connection is established or an old connection is interrupted or completed, such events may affect only a subset of the existing connections. The above observation has been exploited to develop an exact and more efficient max-min fair rate allocation implementation under the assumption of bottleneck links only in the access side of the network. More details about such implementation may be found in [37]. The reported results show that the algorithm proposed in [37] outperforms traditional max-min computation approaches by as much as a factor 100 for a million nodes network. As in [37], we made the assumption that rate bottlenecks occur only in the access part of the network. This assumption is employed in both analytical models appeared in the literature [38] as well as in simulation programs such as [35] and [39]. It is justified by the current bandwidth gap between access links and core network trunk, and by the empirical observation that practical P2P clients typically further throttle the upload bandwidth, which in most cases results fully utilized by the uploading connections.

Obviously, the most serious limit in our approach is that the max-min fair bandwidth allocation well approximates a TCP-like steady state bandwidth sharing. Due to this, our approach is well suited to the case of persistent connections between peers. In addition, it is currently impossible to simulate Transit-Stub topologies such as the ones generated by GT-ITM topology generator. To overcome this last limit, extending the efficient and exact implementation of max-min fair rate allocation proposed in [37] to the case of generic topologies, even if not trivial, could be a reasonable solution.

\subsection{Implementation details}

OPSS is written in $\mathrm{C}++$ and is publicly available [10] under GPL license. It was designed according to a modular implementation logic. Figure 1 illustrates the main blocks of the simulator architecture.

As the figure shows, it is possible to identify three layers:

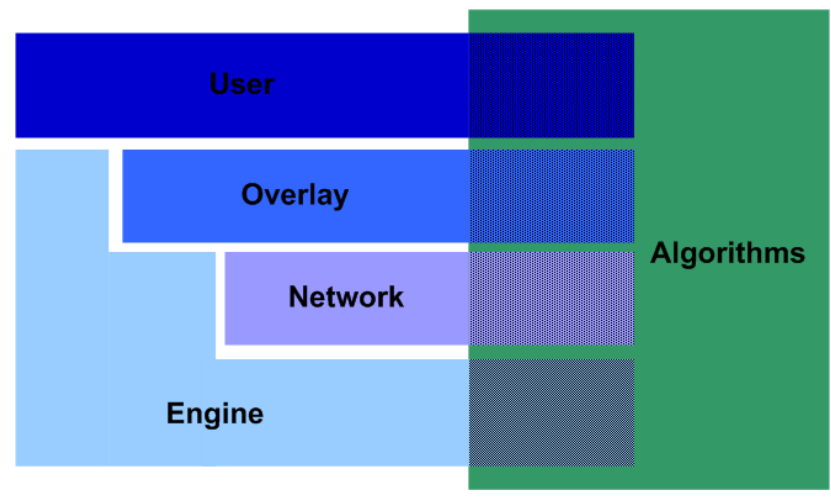

Figure 1: OPSS architecture

User, Overlay and Network. User layer represents the peer behavior, taking into account for example connection and disconnection policies (i.e. the "churn" behavior). Overlay layer is responsible to simulate the overlay network and the overlay interactions between peers. Network layer represents the network behavior, and it currently implements the optimized max-min fair rate allocation approach as described in [37]. All the above layers interact with the Engine block, which contains the discrete-event-related classes and manages the event executions. Engine block is also responsible for output log file where events are dumped with the corresponding time. The set of events that will be included in the log file is customizable to prevent log files to become too big in size. While User, Overlay, Network and Engine include the basic structures common to any $\mathrm{P} 2 \mathrm{P}$ streaming system, the Algorithms block is responsible of the $\mathrm{P} 2 \mathrm{P}$ streaming algorithm and application to be simulated, including the control communication between nodes and the scheduling algorithm of stream segments. It inherits the basic structures of User, Overlay, Network and Engine, and allows to customize them. In such a way, it allows the implementation of any P2P live streaming mechanism.

The previously described approach makes OPSS a very flexible simulator, as it offers the possibility of implementing the logic of $\mathrm{P} 2 \mathrm{P}$ streaming application as separate module. Moreover, simulator users may exploit different basic classes provided by User, Overlay, Network and Engine blocks and potentially implement any kind of P2P streaming algorithms. For further information about how to write algorithms, please refer to the guide available on the reference site.

\subsection{Performance metrics}

In this section we discuss the performance metrics we may evaluate using OPSS. Due to the characteristics of the application (streaming of real-time multimedia flows), the considered metrics are basically related to the delay of the received chunks. In the definition and evaluation of these delay related metrics, we need also to carefully consider that some chunks may not be received by some receiver.

Consider a real time multimedia streaming system where the multimedia stream is divided in chunks of duration $T[\mathrm{~s}]$. The origination of chunks starts at $t=t_{\text {start }}=0$, and ends up at $t=t_{\text {end }}$. The total number of originated chunks will be $t_{\text {end }} / T$. In order to produce consistent measurements, we need to observe the system in an interval of duration 
$w_{\text {end }}-w_{\text {start }}$, with $0 \leq w_{\text {start }}<w_{\text {end }} \leq t_{\text {end }}$. For simplicity, we assume that $w_{\text {start }}$ corresponds to the generation time of one given chunk, and we observe $C$ chunks starting from the one created at $w_{\text {start }}$. Therefore the generation times of the observed chunks will be $t_{c}=w_{\text {start }}+(c-1) T, c=1,2, \cdots, C$. The first observed chunk originates at $t_{1}=w_{\text {start }}$ and the last observed chunk at $t_{C}=w_{\text {start }}+(C-1) T$. In order to allow the last chunk to be received by all receivers, we need to choose our observation interval end time $w_{\text {end }}$ such that $w_{\text {end }}>t_{C}$. In particular, let be $w_{\text {end }}=t_{C}+D_{\max }$, where $D_{\max }$ is the maximum delay we are considering in our evaluation of the system. Note that the origination of chunks will continue also after the origination of the last observed chunk, in the time interval in which we are still observing the system and waiting for the last observed chunk to be received.

Assume that there are $(N-1)$ receiving nodes ${ }^{3}$ and that node $n$ receives the $c$-th observed chunk at time $t_{r}(c, n)=$ $w_{\text {start }}+(c-1) T+d(c, n)$. Then $d(c, n)$ is the delay of chunk $c$ at node $n$. Let us consider the last chunk $C$. If $d(C, n)>D_{\max }$, the event is out of our observation window and it will be lost. For the generic chunk $c$, the relative reception event at node $n$ goes out of our observation window if $d(c, n)>D_{\max }+(C-c+1) T$. From a methodological point of view, it is not good that the maximum observable delay for a chunk depends on the chunk number $c$. Therefore we think it should be better to set $D_{\max }$ as maximum chunk delay for all chunks and to consider a chunk $c$ lost if $d(c, n)>$ $D_{\max }$.

We consider that a receiving node $n$ can be active or not by defining its activity function $a(t, n)$ as follows: $a(t, n)=1$ if node $n$ is active at time $t, a(t, n)=0$ if node $n$ is not active at time $t$. The activity of node $n$ during the observation window is:

$$
A(n)=\frac{1}{w_{\text {end }}-w_{\text {start }}} \int_{w_{\text {start }}}^{w_{\text {end }}} a(t, n) d t
$$

The number of active nodes at time $t$ is given by:

$$
N_{A}(t)=\sum_{n} a(t, n)
$$

Therefore the average number of active nodes $N_{A}$ over the observation window will be:

$$
N_{A}=\frac{1}{w_{\text {end }}-w_{\text {start }}} \int_{w_{\text {start }}}^{w_{\text {end }}} N_{A}(t) d t
$$

We define the chunk delivery ratio (CDR) for a chunk $c$ (that depends on $D_{\max }$ ) as the ratio between the nodes that have received the chunk $c$ and the average number of active nodes when the chunk is originated. Note that we should consider the average number of active nodes in a time interval following the chunk origination event as this is the number of potential receivers for the chunk. As the chunk delivery delay is variable, it is not clear over which time interval we should average $N_{A}(t)$. A simpler solution is to define a "conventional" chunk delivery ratio for a chunk $c$ using the overall average number $N_{A}$ of active nodes over the observation window. This is reasonable if the average number of active nodes does not change over time. According to such assumption, the chunk delivery ratio relative to

${ }^{3}$ The total node number is $N$ if we add the stream source to the receiving nodes. chunk $c$ is:

$$
C D R_{D_{\max }}(c)=\frac{1}{N_{A}} \sum_{n} r_{D_{\max }}(c, n)
$$

where $r_{D_{\max }}(c, n)=1$ if chunk $c$ is received by node $n$ with $d(c, n) \leq D_{\max }$, while $r_{D_{\max }}(c, n)=0$ if chunk $c$ either is not received or is received with $d(c, n)>D_{\max }$.

We also define the overall chunk delivery ratio (that depends on $D_{\max }$ as well) as:

$$
C D R_{D_{\max }}=\frac{1}{C} \sum_{c} C D R_{D_{\max }}(c)=\frac{1}{C \cdot N_{A}} \sum_{c, n} r_{D_{\max }}(c, n)
$$

Metrics related to the chunk delivery ratio are very common in $\mathrm{P} 2 \mathrm{P}$ streaming performance studies [8][9]. These metrics can measure the continuity of the playback; however, to better characterize live streaming applications, it is advisable to take into account also the absolute delay of chunks at the peer nodes. To this purpose, OPSS allows also to evaluate metrics related to $d(c, n)$, that represents the time interval between the time instant at which chunk $c$ is available at stream source and the time instant at which node $n$ completes the download of chunk $c$. Specifically, we consider the average chunk delay for a chunk $c$, which can be evaluated by averaging the delay over all nodes that received that chunk (with a delay lower than or equal to $D_{\max }$ ):

$$
\bar{d}_{D_{\max }}^{\text {chunk }}(c)=\frac{1}{N_{A} \cdot C D R_{D_{\max }}(c)} \sum_{n \mid r_{D_{\max }}(c, n)=1} d(c, n)
$$

It could be interesting to consider the perspective of a given node $n$ and to evaluate the perceived performances. First of all, we can evaluate the chunk delivery ratio seen by a given node $n$ :

$$
C D R_{D_{\max }}^{\text {node }}(n)=\frac{1}{C \cdot A(n)} \sum_{c} r_{D_{\max }}(c, n)
$$

We can also evaluate the average chunk delay perceived by a generic node $n$ :

$$
\bar{d}_{D_{\max }}^{\text {node }}(n)=\frac{1}{C \cdot A(n) \cdot C D R_{D_{\max }}^{\text {node }}(n)} \sum_{c \mid r_{D_{\max }}(c, n)=1} d(c, n)
$$

It is also interesting to consider the $\alpha$-chunk delay percentile (CDP) of the distribution of chunk delay perceived by a generic node $n$. Typical values that we can consider are $\alpha=95, \alpha=99$.

$$
C D P_{D_{\max } \text { made }}(\alpha, n)=x \mid \operatorname{Prob}\{d(c, n)<x\}=\frac{\alpha}{100}
$$

The overall average chunk delay can be evaluated by averaging the delay over all received chunks (with a delay lower than or equal to $\left.D_{\max }\right)$ :

$$
\bar{d}_{D_{\max }}=\frac{1}{C \cdot N_{A} \cdot C D R_{D_{\max }}} \sum_{c, n \mid r_{D_{\max }}(c, n)=1} d(c, n)
$$

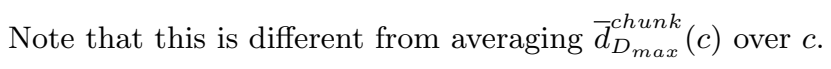




\section{THE EVALUATED P2P STREAMING AL- GORITHMS}

Goal of this section is to show OPSS performance in terms of both scalability and capability of producing correct results. To this purpose, we simulated two "trivial" P2P streaming distribution schemes ("Balanced $M$-ary tree" and "Trivial Mesh"), for which we could easily derive analytical models. We compared the experimental results achieved by OPSS with the results of the analytical model, and verified OPSS correctness. On the other hand, OPSS scalability was simply evaluated by simulating an ever increasing number of nodes. In the following subsections, we describe the simulated distribution schemes and we report the corresponding analytical models and experimental results.

\subsection{Balanced $M$-ary tree}

This stream distribution scheme corresponds to a balanced $M$-ary distribution tree. The stream source is the root of the tree. The stream video is divided into segments or chunks, and $R=1 / T$ denotes the source chunk rate [chunk/s]. Each node downloads chunks from one single node, and it uploads chunks to $M$ nodes. According to the tree graphs jargon, each node has $M$ children. We assume that all nodes (including the stream source) join the system simultaneously and form the distribution tree. We also assume a static situation, in which all nodes persist through the whole lifetime of the simulation.

Due to the last assumption, the issues related to the number of active nodes at a time instant and the average number of active nodes may be neglected. Another assumption we make is that $w_{\text {start }}=t_{1}=0$, where according to subsection $3.4 w_{\text {start }}$ and $t_{1}$ denote respectively the observation start time and the first chunk creation time. The $c$-th chunk will be referred to as $c$ and the relative creation time is $t_{c}=(c-1) / R$.

We now introduce the level concept. With reference to a node, the level $l$ represents its distance from stream source as number of hops in the overlay tree. The level of the stream source is $l=0$, while the last level is denoted as $l=L$. If all levels are complete, the number of nodes at level $l$ is $M^{l}$ and the total number of nodes is $N=\sum_{l=1}^{L} M^{l}$. In the following, we will always consider trees with complete levels.

All nodes are assigned an access link with uplink and downlink capacities $W_{u p}$ and $W_{\text {down }}[$ chunk/s]. To simplify, we assume symmetrical access links, that means also $W=W_{u p}=W_{\text {down }}$. As consequence, each node downloads chunks at $W / M[\mathrm{chunk} / \mathrm{s}]$ from its father in the tree. If $W / M<R$, the distribution system cannot work as each node does not have enough capacity to download the stream of chunks. We thus restrict our attention to the case in which the available portion of the father's uplink capacity is greater than or equal to the rate of the stream to be received: $W / M \geq R$.

It is convenient to express the delay of chunk $c$ at node $n$ in terms of the corresponding node level $l$. The reason is that all nodes at the same level perceive the same delay. Specifically, given the level $l, l=1,2, \cdots, L$, and the chunk $c$, the corresponding chunk delay is

$$
d(c, l)=\frac{M}{W} \cdot l
$$

The average chunk delay for chunk $c$ is

$$
\begin{aligned}
\bar{d}^{\text {chunk }}(c) & =\frac{\sum_{l=1}^{L} M^{l} \cdot d(c, l)}{\sum_{l=1}^{L} M^{l}} \\
& =\frac{M\left\{M^{l}[L(M-1)-1]+1\right\}}{W(M-1)\left(M^{l}-1\right)}
\end{aligned}
$$

Note that it does not depend on $c$. The reference to $D_{\max }$ is omitted, as we are assuming an ideal system where chunks are not lost. We only need to set $D_{\max }>L \cdot W / M$ so that we are able to observe all chunk reception events.

The average chunk delay perceived by a generic node at level $l$ is

$$
\bar{d}^{\text {node }}(l)=\frac{1}{C} \sum_{c=1}^{C} d(c, l)=\frac{M}{W} \cdot l
$$

The assumption in (13) is that $C$ chunks are observed during the observation window.

Let us now consider the experimental results we achieved by setting $M=2$. In more detail, we simulated a set of binary distribution trees by varying the maximum depth $L$ of the tree in the range $[1,17]$ (e.g. the node number ranges from 3 to 131171). Figure 2 illustrates the binary tree corresponding to $L=4$. Observation time end was set to $3600 \mathrm{sec}$. Moreover, $W=2[\mathrm{chunk} / \mathrm{s}$ ] and $R=1$ [chunk/s] were used as upload capacity and chunk creation rate. We highlight the "deterministic" nature of the simulations, since there is no variability in the input data and all nodes are always active. Therefore, the results of each simulation are relative to a single simulation run and we do not indicate any confidence interval (the same considerations apply to the next subsection).

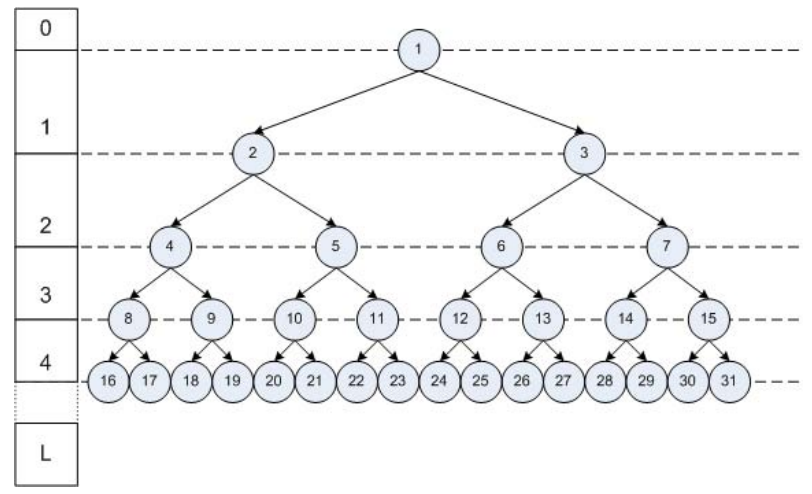

Figure 2: Binary distribution tree with $\mathrm{N}=31$ nodes

Figure 3 shows the chunk delivery ratio $C D R(c)$ defined in (4) versus $c$. As the observation end time $w_{\text {end }}$ is $3600 \mathrm{sec}$ and the last observed chunk is generated at 3600 , we are not able to observe the complete diffusion of all chunks created during the observation window. The correct approach in order to observe all reception events is set $C$ to the greatest $c$ such that the condition $(L+c-1) M / W<w_{\text {end }}$ is verified. For instance, when the simulated nodes are 255, solving the above condition leads to $c=3593$, corresponding to the highest vertical step in the curve for 255 nodes.

The cumulative distribution function of the average chunk delay for a given chunk $\bar{d}^{\text {chunk }}(c)$, defined in (12), is illustrated in Figure 4. According to equation (12), the cumulative distribution function confirms that the average chunk 


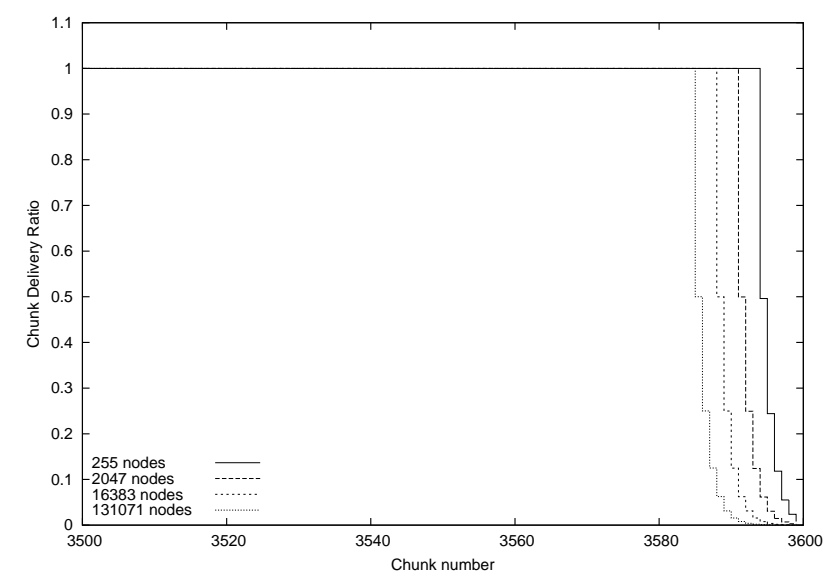

Figure 3: Chunk delivery ratio

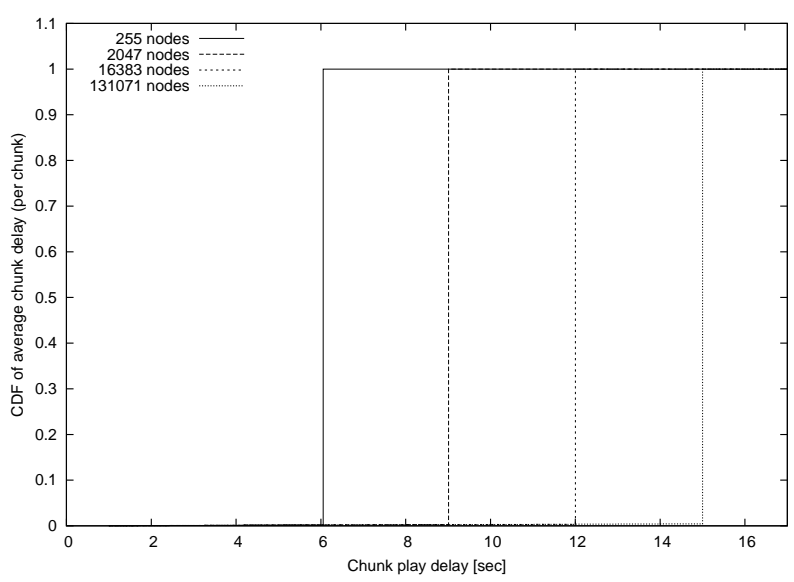

Figure 4: Cumulative distribution function of average chunk delay for a given chunk

delay is constant, and there is a perfect matching between the values achieved by equation (12) and the values achieved by simulation.

Figure 5 shows the cumulative distribution function of the average chunk delay perceived by the generic node. Given a number of simulated nodes, the steps correspond to the different tree levels and their probability values may be deduced from the ratio between the number of nodes in that level and the total number of nodes.

\subsection{Trivial mesh}

The algorithm represents a really simple streaming distribution system based on mesh topologies. Specifically, the stream source generates chunks at rate $R$ [chunk/sec] and it uploads $S$ chunks simultaneously to $S$ nodes. All other nodes (i.e. excluding the source node) maintain $S$ connections for downloading chunks and $S$ connections for uploading chunks, and they use them simultaneously to upload/download different chunks. Thus nodes form groups of $S$ nodes. The first $S$ nodes are connected directly with stream source and download the stream chunks from it. The second group of $S$ nodes opens a connection with each node of the first group to download available chunks, and so on. In such a way, if we further assume that the total number of

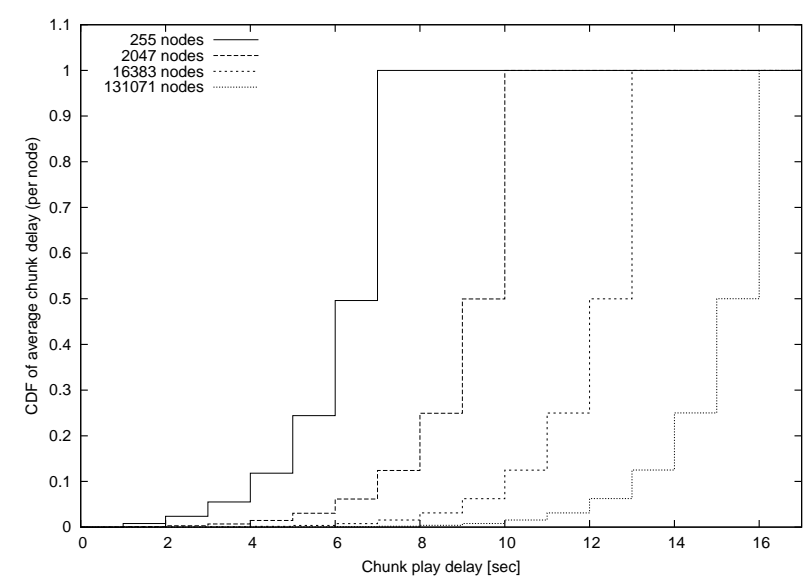

Figure 5: Cumulative distribution function of average chunk delay at a given level

nodes is $N=L \cdot S+1$, it is possible to identify $L$ different levels, each consisting of a group of $S$ nodes. The previous assumption implies also that all levels are complete. Figure 6 shows the trivial mesh topology corresponding to $N=17$ and $S=4$.

Building the overlay topology according to the above trivial mesh scheme is not smart, as the underlying algorithm does not allow to increase the number of peers at each level: the tree depth increases linearly with the node number and the chunk transfer delay increases as well. Anyway, the purpose of our work now is just to check OPSS capability of producing the expected results, and we are going to provide an analytical model of this trivial mesh. Like in the previous experiment, we assume that nodes persist through the whole simulation time. Moreover, with regard to access link upload and download capacities, the condition $W=W_{u p}=W_{\text {down }}$ [chunk/s] holds. There is however one exception: the stream source has a capacity that is $S$ times the capacity of other nodes. This means that the $S$ nodes connected to the stream source download chunks at rate $W$. The other nodes may download $S$ chunks in parallel from $S$ different suppliers, and each chunk is downloaded at a rate of $W / S$ [chunk/s]. We will focus on the case $W=R$.

Since all nodes in a level perceive the same chunk delay, we can refer to levels instead of individual nodes. With re-

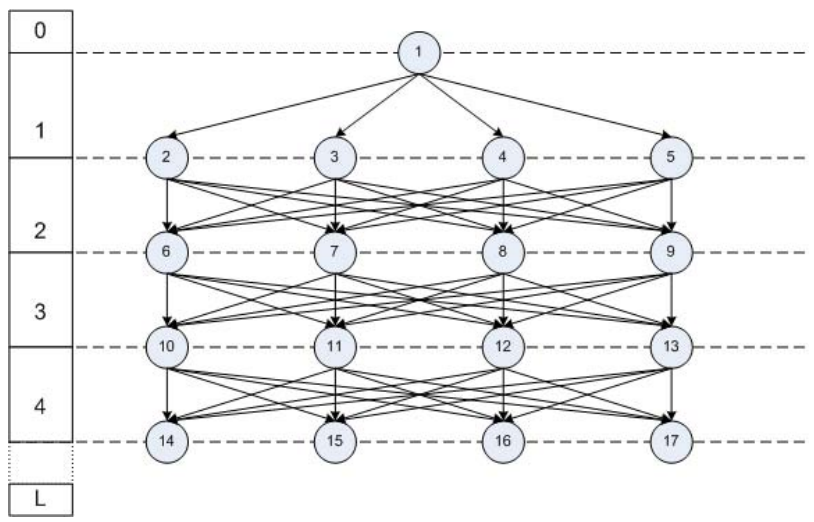

Figure 6: Trivial mesh with $\mathrm{N}=17$ nodes and $\mathrm{S}=4$ 
gard to the observation window, we assume that i) $w_{\text {start }}=$ $t_{1}=0$, ii) the creation time of the generic c-th chunk is $t_{c}=(c-1) / R$. In addition, we assume that source node and nodes at level 1 join the system at time $w_{\text {start }}$, while all other nodes join the system simultaneously at time instant $t_{S}=S / R$, i.e. when the first $S$ chunks have already been created and transferred to nodes at level 1. Therefore, at instant $t_{S}$ each node of level 2 will open $S$ connections to download the first $S$ chunks from the $S$ nodes at level 1. The download of these $S$ chunks will last $S / W[\mathrm{~s}]$. As in our hypothesis, the downloads will last exactly $S / R$ or equivalently $S T[\mathrm{~s}]$. When such downloads end, there will be $S$ new chunks available at nodes at level 2 and nodes at level 3 will start $S$ new downloads from nodes at level 2 . This procedure is straightforwardly replicated in all levels below.

Given the level $l, l=1,2, \cdots, L$, and the chunk $c$, the corresponding chunk delay is

$$
d(c, l)=\frac{S}{R}+\left[\operatorname{ceil}\left(\frac{c}{S}\right)+l-1\right] \frac{S}{W}-\frac{c-1}{R}
$$

This delay will be periodic of period $S$, as a burst of $S$ chunks will be received at the same time by nodes at same level. In particular, the most recent chunk of the burst will experience the lowest delay, the oldest chunk of the burst will experience the highest delay and the delay difference among two next chunks in the burst is $T$.

The average chunk delay for the chunk $c$ is

$$
\begin{aligned}
\bar{d}^{\text {chunk }}(c) & =\frac{\sum_{l=1}^{L} S \cdot d(c, l)}{\sum_{l=1}^{L} S} \\
& =\frac{S}{2 W}(L-1)+\frac{S}{W} \operatorname{ceil}\left(\frac{c}{S}\right)-\frac{c-1-S}{R}
\end{aligned}
$$

The average chunk delay perceived by a generic node at level $l$ is evaluated under the assumption that the number $C$ of observed chunks is an integer multiple of $S$, e.g. $C=J \cdot S$ with $J \in Z^{+}$. Specifically, it results:

$$
\begin{aligned}
\bar{d}^{\text {node }}(l) & =\frac{1}{C} \sum_{c=1}^{C} d(c, l) \\
& =\frac{S[R(J-1+2 l)-W(J-1)]}{2 R W}
\end{aligned}
$$

At this point we report the experimental results we achieved by setting $S=4$. The node number ranges from 101 to 3601 passing through 1001 and 3401. Observation end time was set to $w_{\text {end }}=3600$. Moreover, $W=R=1[$ chunk $/ \mathrm{s}]$.

The chunk delivery ratio is shown in Figure 7 . According to formula (16), the average chunk delay at level $l$ is linearly dependent of $l$. This means that, due to the fixed observation window, when the node number and consequently the level number grow, an ever increasing number of chunk reception events will be lost. When the node number is 3601 , the first created chunk is received only by nodes in the first $(L-1)$ levels. In reason of this, simulating a higher node number does not make sense if the observation window is not accordingly increased.

The cumulative distribution of the average chunk delay for a given chunk is illustrated in Figure 8. As it can be seen, each curve is characterized by two different parts: the first one grows almost linearly and accounts for the chunks that are not received by all nodes within the observation window; the second one relates to the chunks that all nodes receive

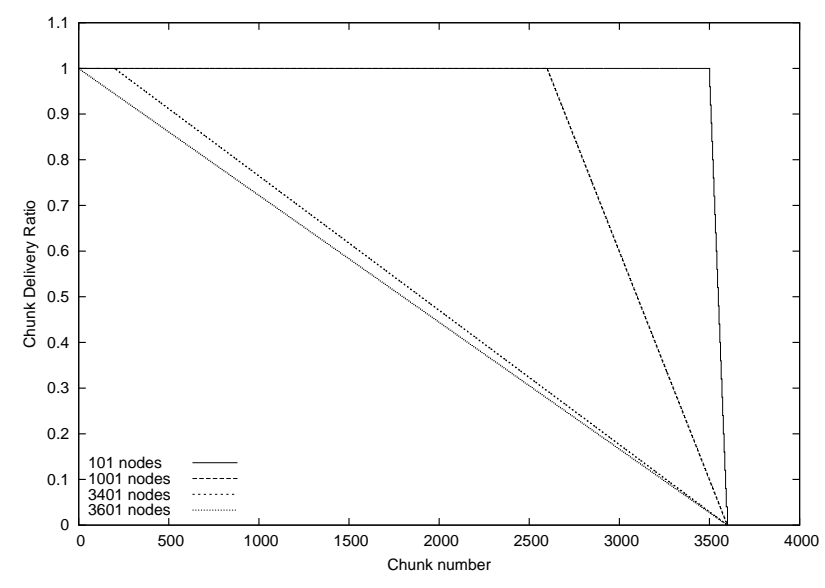

Figure 7: Chunk delivery ratio

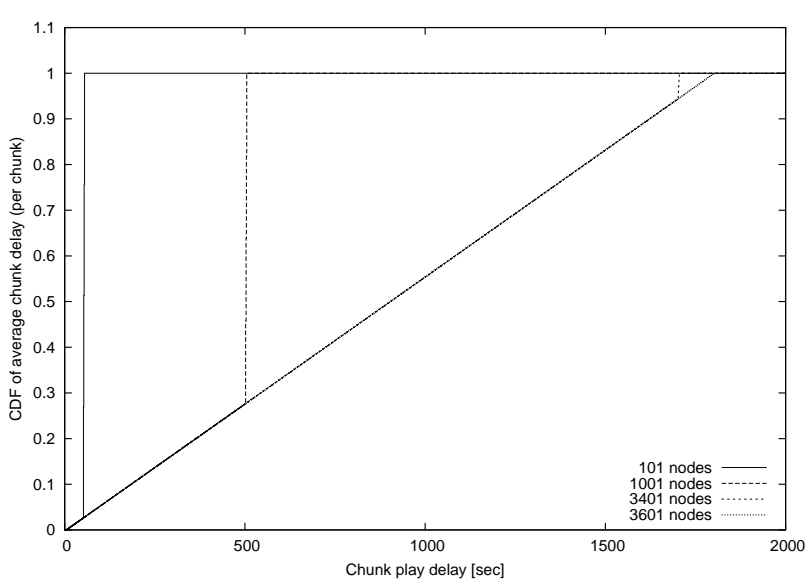

Figure 8: Cumulative distribution function of average chunk delay for a given chunk

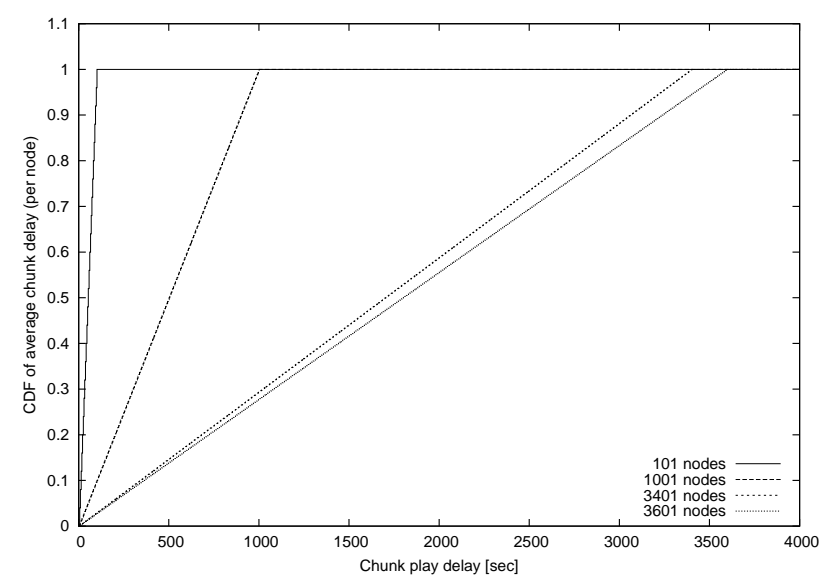

Figure 9: Cumulative distribution function of average chunk delay at a given level

within the observation window and it is almost vertical (actually there are $S$ different values separated by 1 second, as the average chunk delay is periodic with period $S$ and the values it may take increase by step $T=1 / R$ ). 
Figure 9 shows the cumulative distribution function of average chunk delay at a given level. The reported curves exhibit a step trend with each step corresponding to a specific level, even if it is difficult to appreciate the step trend, as the node number and consequently the level number grow. This complies with equation (16), even if the last one has been derived under the approximation of observed chunk number multiple of $S$.

\subsection{Simulator performance}

Just to give an idea about OPSS performance, we provide some details about the simulation computational load on the pc-desktop we used for our experiments. Specifically, the pc was equipped with a $3.2 \mathrm{GHz}$ bi-processor $\mathrm{CPU}$ and 4 Gigabyte RAM. Table 1 refers to the binary tree algorithm and shows, for a given node number, the time necessary to complete the simulation, the resulting log file size and the time necessary to analyze log file with an our own $\mathrm{C}++$ application. The analysis results have been then used to achieve the previously reported graphs.

\begin{tabular}{|l|l|l|l|}
\hline \multicolumn{4}{|c|}{ Binary tree algorithm } \\
\hline Nodes & Simulation time & Log file size & Analysis time \\
\hline 131071 & $\sim 6 h$ & $15.6 \mathrm{~GB}$ & $\sim 5 h$ \\
\hline
\end{tabular}

Table 1: Main computational load parameters

\section{CONCLUSIONS}

In this paper we have presented OPSS, a tool for the simulation of large-scale $\mathrm{P} 2 \mathrm{P}$ streaming networks, and we tested it by using two reference algorithms for building a live streaming $\mathrm{P} 2 \mathrm{P}$ distribution system. The simulator has produced consistent results, perfectly aligned with the analytical models of the reference algorithms. Our current works concern first the extensions to the network part of the simulator, in order to consider more complex topology with respect to the "bottleneck-in-the-access" approach that is currently implemented. The second direction of work is to use OPSS for performance evaluation of mesh-based algorithms for a live streaming $\mathrm{P} 2 \mathrm{P}$ distribution systems. As we have shown that there are no tools allowing to make this evaluation for large scale $\mathrm{P} 2 \mathrm{P}$ systems and considering the problem of resources utilization at network transport level, we believe that OPSS will be able to provide interesting insights on the problem.

\section{REFERENCES}

[1] C. Diot, B. Levine, B. Lyles, H. Kassem, D. Balensiefen, Deployment issues for the IP multicast service and architecture, in IEEE Network, vol. 14 (1), 10-20, 2000

[2] Y. Chu, S. G. Rao, H. Zhang, A case for end system multicast, in Proceedings of ACM SIGMETRICS 2000, Santa Clara, CA,USA, 2000

[3] B. Zhang, S. Jamin, L. Zhang, Host multicast: a framework for delivering multicast to end users, in Proceeding of IEEE INFOCOM, New York, NY, USA, 2002

[4] S. Banerjee, B. Bhattacharjee, C. Kommareddy, Scalable application layer multicast, in Proceedings of ACM SIGCOMM, Pittsburgh, PA, USA, 2002
[5] D. A. Tran, K. A. Hua, T. Do, ZIGZAG: an efficient peer-to-peer scheme for media streaming, in Proceedings of IEEE INFOCOM, San Francisco, CA, USA, 2003

[6] V. N. Padmanabhan, H. J. Wang, P. A. Chou, Distributing streaming media content using cooperative networking, in Proceedings of NOSSDAV, Miami Beach, FL, USA, 2002

[7] M. Castro, P. Druschel, A. Kermarrec, A. Nandi, A. Rowstron, A. Singh, SplitStream: high-bandwidth multicast in cooperative environments, in Proceedings of the 19th ACM Symposium on Operating Systems Principles (SOSP'03), The Sagamore, Bolton Landing, NY, USA, 2003

[8] X. Zhang, J.C. Liu, B. Li, P. Yum, CoolStreaming/DONet: A data-driven overlay network for efficient live media streaming, in Proceedings of IEEE INFOCOM, Miami, FL, USA, 2005

[9] M. Zhang, L. Zhao, Y. Tang, J. Luo, S. Yang, Large-Scale Live Media Streaming over Peer-to-Peer Networks through Global Internet, in Proceedings of ACM Multimedia 2005, Singapore, Singapore, 2005

[10] OPSS simulator, http://netgroup.uniroma2.it/ twiki/bin/view.cgi/Netgroup/OpssPublicPage

[11] A.J. Ganesh, A.M. Kermarrec, L. Massoulie, Peer-to-peer membership management for gossip-based protocols, in IEEE Transactions on Computers, 52(2), 2003

[12] PlanetLab testbed, http://www.planet-lab.org/

[13] B. Chun, D. Culler, T. Roscoe, A. Bavier, L. Peterson, M. Wawrzoniak, M. Bowman, PlanetLab: An Overlay Testbed for Broad-Coverage Services, in ACM Computer Communications Review, vol. 33, no. 3, 2003

[14] M. Zhang, Y. Xiong,, Q. Zhang, S. Yang, On the Optimal Scheduling for Media Streaming in Data-driven Overlay Networks, in Proceedings of IEEE Globecom 2006, San Francisco, CA, USA, 2006

[15] V. Pai, K. Kumar, K. Tamilmani, V. Sambamurthy, A.E. Mohr, Chainsaw: Eliminating Trees from Overlay Multicast, in Proceedings of 4th International

Workshop on Peer-to-Peer Systems (IPTPS'05), Ithaca, NY, USA, 2005

[16] K. Calvert, E. Zegura, S. Bhattacharjee, How to Model an Internetwork, in Proceedings of IEEE INFOCOM, San Francisco, CA, USA, 1996

[17] PPLive web site, www.pplive.com/en/index.html

[18] SopCast web site, http://www.sopcast.org/

[19] X. Hei, C. Liang, J. Liang, Y. Liu, K. Ross, Insights into PPLive: A Measurement Study of a Large-Scale P2P IPTV System, in Proceedings of IPTV Workshop, International World Wide Web Conference, Edinburgh, Scotland, 2006

[20] S. Ali, A. Mathur, H. Zhang, Measurement of Commercial Peer-To-Peer Live Video Streaming, in Proceedings of Workshop on Recent Advances in P2P Streaming, Waterloo, ON, Canada, 2006

[21] Aurora simulator, http: //freenet.cvs.sourceforge.net/freenet/aurora/

[22] Serapis simulator, http: //freenet.cvs.sourceforge.net/freenet/Serapis/

[23] T. M. Gil, F. Kaashoek, J. Li, R. Morris, J. Stribling, P2Psim simulator, 
http://pdos.csail.mit.edu/p2psim/

[24] Rice University, FreePastry simulator, http://freepastry.org/FreePastry/download.html

[25] I. Stoica, M. Walfish, Chord simulator, http://cvs.pdos.csail.mit.edu/cvs/ checkout $\sim /^{\sim}$ sf snet/simulator/

[26] Neurogrid simulator, http://www . neurogrid.net/php/simulation.php

[27] S. Joseph, An extendible open source P2P simulator, P2P Journal, 1-15, 2003

[28] N. S. Ting, R. Deters, 3LS - A Peer-to-Peer network simulator, in Proceedings of 3rd International Conference on Peer-to-Peer Computing, Linköping, Sweden, 2003

[29] M. Jelasity, G. P. Jesi, A. Montresor, S. Voulgaris, PeerSim simulator, http://peersim.sourceforge.net/

[30] M. Jelasity, A. Montresor, O. Babaoglu, A modular paradigm for building self-organizing peer-to-peer applications, in Proceedings of the International Workshop on Engineering Self-Organising Applications (ESOA'03), Melbourne, Australia, 2003

[31] Q. He, M. Ammar, G. Riley, H. Raj, R. Fujimoto, GnutellaSim simulator, http://www-static.cc. gatech.edu/computing/compass/gnutella/

[32] Q. He, M. Ammar, G. Riley, H. Raj, R. Fujimoto, Mapping peer behavior to packet-level details: a framework for packet-level simulation of Peer-to-Peer systems, in Proceedings of MASCOTS 2003, Orlando (FL), USA, 2003

[33] The Network Simulator ns-2, http://www.isi.edu/nsnam/ns/

[34] D. Bertsekas, R. Gallager, Data Networks, Prentice Hall, Englewood Cliffs, NJ, 1987

[35] M.Baker, T. Giuli, Narses: a scalable flow-based network simulator, Technical report, Stanford University, 2002, available on line at http: //arxiv.org/PS_cache/cs/pdf/0211/0211024v1.pdf

[36] W. Yang, N. Abu-Ghazaleh, GPS: A General Peer-to-Peer Simulator and its Use for Modeling BitTorrent, in Proceedings of MASCOTS 2005, Atlanta, GA, September 2005

[37] F. Lo Piccolo, G. Bianchi, S. Cassella, Efficient simulation of bandwidth allocation dynamics in P2P Networks, in Proceedings of Globecom 2006, San Francisco, CA, USA, 2006

[38] D.Qiu, R.Srikant, Modeling and Performance Analysis of BitTorrent-Like Peer-to-Peer Networks, in Proceedings of ACM SIGCOMM'04, Portland, OR (USA), September 2004

[39] M. Schlosser, T. Condie, S. Kamvar, Simulating a P2P file-sharing network, in Proceedings of 1st Workshop on Semantics in P2P and Grid Computing, Budapest, Hungary, May 2003 